\title{
Biogeochemical processes responsible for iron duricrust cementation
}

E. GAGEN ${ }^{1,2 *}$, A. PAZ ${ }^{1}$, A. LEVETT ${ }^{1}$, G. SOUTHAM ${ }^{1}$

${ }^{1}$ School of Earth and Environmental Sciences, The University of Queensland, St Lucia, QLD 4072, Australia

(e.gagen@uq.edu.au)

${ }^{2}$ Australian Centre for Ecogenomics, School of Chemistry and Molecular Biosciences, The University of Queensland, St Lucia, QLD 4072, Australia

High-grade iron ore deposits in tropical areas are capped by a hard, erosion-resistant iron duricrust that protects the friable ore below it and supports a unique above-ground ecosystem. Combining microscopy, molecular biology, cultivation and geochemical analyses on duricrust samples from Carajás, Brazil, we confirmed the critical role microorganisms play in the ongoing goethite dissolution and re-precipitation that leads to natural stabilisation of these duricrusts. Using microbial cultures enriched from iron duricrust environments, we have demonstrated that microbially-driven consolidation and stabilisation of crushed duricrust material, is possible at laboratory and pilot-scale. A goethite-reducing enrichment culture was key in slope stabilisation of crushed duricrust material at laboratory scale and a metagenome assembled genome of a novel, fermentative iron-reducing Telmatospirillum spp. was recovered from the culture. Fermentation-driven iron reduction was also key in $\mathrm{m}^{3}$ pilotscale experiments, which led to aggregation of crushed iron duricrust material producing $\sim 20 \times 20 \times 20 \mathrm{~cm}$ aggregates. Candidate phyla radiation (CPR) microorganisms dominated the microbial community in that system. These data highlight the importance of coupling traditional approaches with emerging molecular biology techniques in geomicrobiology. Furthermore, our laboratory and pilot scale findings demonstrate the potential for harnessing geomicrobiological processes for stabilisation of mine waste and accelerated remediation of tropical iron ore mine areas. 